\title{
Experimental verification of boundary conditions for numerical simulation of airflow in a benchmark ventilation channel
}

\author{
Frantisek Lizal ${ }^{1, a}$, Pavel Niedoba ${ }^{1}$, Libor Seda ${ }^{1}$, Jan Jedelsky ${ }^{1}$, Miroslav Jicha ${ }^{1}$ \\ ${ }^{1}$ Brno University of Technology, Faculty of Mechanical Engineering, Energy Institute, Technicka 2896/2, Brno 616 69, Czech Republic
}

\begin{abstract}
Correct definition of boundary conditions is crucial for the appropriate simulation of a flow. It is a common practice that simulation of sufficiently long upstream entrance section is performed instead of experimental investigation of the actual conditions at the boundary of the examined area, in the case that the measurement is either impossible or extremely demanding. We focused on the case of a benchmark channel with ventilation outlet, which models a regular automotive ventilation system. At first, measurements of air velocity and turbulence intensity were performed at the boundary of the examined area, i.e. in the rectangular channel $272.5 \mathrm{~mm}$ upstream the ventilation outlet. Then, the experimentally acquired results were compared with results obtained by numerical simulation of further upstream entrance section defined according to generally approved theoretical suggestions. The comparison showed that despite the simple geometry and general agreement of average axial velocity, certain difference was found in the shape of the velocity profile. The difference was attributed to the simplifications of the numerical model and the isotropic turbulence assumption of the used turbulence model. The appropriate recommendations were stated for the future work.
\end{abstract}

\section{Introduction}

Computational fluid dynamics (CFD) is an efficient tool, which is used in many applications for calculation of airflow characteristics. In a frame of broader research task, we investigate the air flow field downstream of a benchmark automotive vent. The research is performed both numerically and experimentally. At first, the experimental test rig was designed, and the geometry of the channel was modelled in a simplified fashion and is used for the numerical simulation. The main goal of the broader project is to determine the influence of the upstream channel, adjustable grilles and design of the closing valve (these parts are hereafter referred to as the main section) on the flow.

The precise setting of boundary conditions at the inlet of the critical section, namely velocity profile and turbulence intensity, is necessary for a correct simulation of the flow. There are two ways to determine the boundary conditions. The first option is the experimental measurement performed directly upstream of the main section, the alternative way is the numerical simulation of a sufficiently long entrance section.

In this paper we compare the results acquired experimentally using constant temperature anemometry and numerically at the inlet boundary of the main section to determine the possible error in the velocity and turbulence intensity settings.

\section{Methods}

The whole test rig for measurement of the flow downstream of the benchmark automotive vent (Figure 1) consists of a regular automotive ventilator connected to a $12 \mathrm{~V}$ laboratory electric supply. The flow rate is calculated from a measured pressure loss of an orifice plate according to EN ISO 5167. 3D fittings and stilling pipes are located upstream and downstream of the orifice.

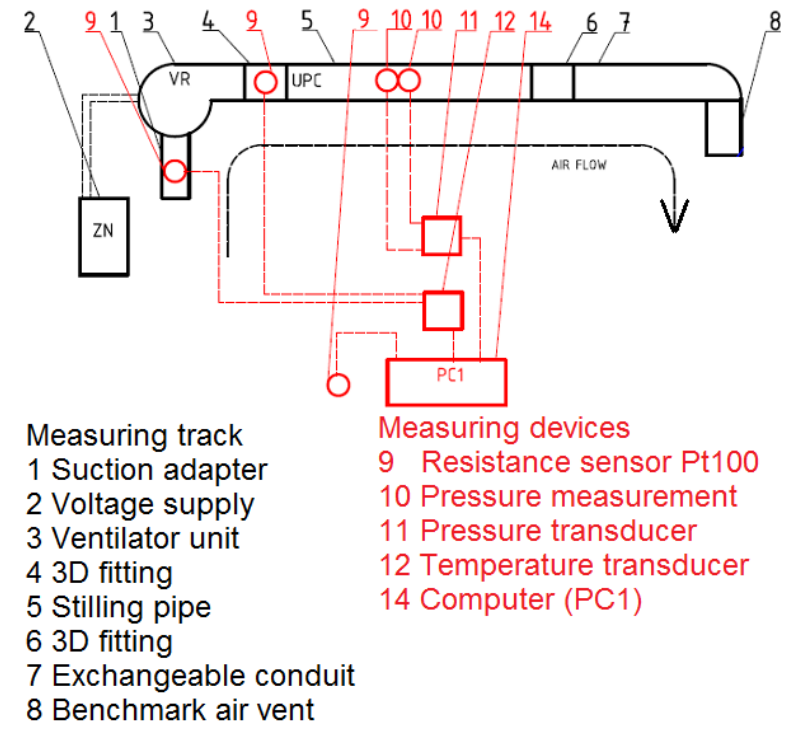

Figure 1. Schematic of the benchmark automotive vent measuring rig [1].

\footnotetext{
${ }^{\mathrm{a}}$ Corresponding author: lizal@fme.vutbr.cz
} 
The conduit directly upstream of the benchmark vent is interchangeable and either straight or bent channel can be inserted. Temperature is measured upstream and downstream of the ventilator and in the surrounding air. Absolute pressure is measured upstream of the orifice and atmospheric pressure is measured in the surroundings.

The velocity and turbulence measurement at the boundary of the main section (upstream of the interchangeable conduit) was performed in three vertical and five horizontal lines $272.5 \mathrm{~mm}$ upstream of the upper edge of the benchmark vent (see Figure 2). Both the measurement and simulation were performed for flowrate of $87.7 \mathrm{~m}^{3} / \mathrm{h}$. Dimensions of the rectangular channel in the measuring cross-section was $42 \times 95 \mathrm{~mm}$. Reynolds number was approximately 18,000, therefore the turbulent velocity profile had been expected.

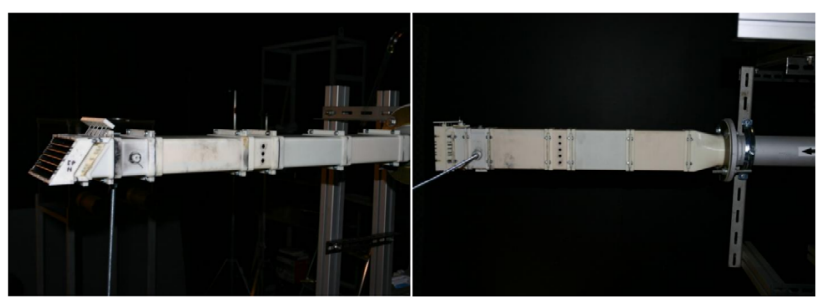

Figure 2. Photographs of the measuring rig (side view on the left and bottom view on the right) with drilled holes for insertion of the velocity probe [1].

\subsection{Numerical simulation}

The three-dimensional incompressible steady-state numerical simulation was carried out with the opensource code OpenFOAM. The governing Reynoldsaveraged Navier-Stokes (RANS) equations were closed with the $k-\omega$ SST turbulence model [2]. The numerical code is based on the finite volume method using the SIMPLE scheme for the incompressible steady-state flow.

Figure 3 shows the simplified numerical model of the benchmark vent. In the process of the simplification, some geometry details had to be neglected to reduce the computational costs. The mesh was generated using the open-source mesh generator SALOME and consists of $1,158,134$ tetrahedral cells. The case requires initial and boundary conditions settings for all the involved fields, i.e. velocity $U$ or volumetric flowrate $V$, pressure $p$, turbulent kinetic energy $k$ and specific rate of dissipation $\omega$. The inlet turbulence properties were set to $k=2.92$ $\mathrm{m}^{2} \mathrm{~s}^{-2}$ and $\omega=637 \mathrm{~s}^{-1}$.

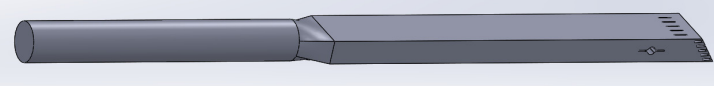

Figure 3. Simplified numerical model of the benchmark vent.

The calculation of the turbulence intensity $(T u)$ from numerical data was based on the turbulent kinetic energy, i.e.

$$
T u=\frac{\sqrt{\frac{2}{3} k}}{\bar{U}} \cdot 100(\%),
$$

where $\sqrt{\frac{2}{3} k}$ represents the root mean square value of the turbulent velocity field $\mathrm{u}$ '. Note that the turbulent kinetic energy assumes isotropic fluctuations, which does not comply with our case of channel flow, where the axial turbulent velocity component dominates. Hence, the difference between numerical and experimental values of turbulent intensity is expected, i.e. the numerical turbulent intensity underestimates the experimental turbulent intensity in the axial direction.

\subsection{Experiments}

The measurement of velocity was performed by Constant Temperature Anemometry (CTA) system Dantec Streamline with single wire probe 55P04 (Dantec Dynamics A/S, Skovlunde, Denmark). The velocity calibration was performed in the range of 0.5 to $20 \mathrm{~m} / \mathrm{s}$ in the Dantec StreamLine ${ }^{\circledR}$ Pro Automatic calibrator. Measuring frequency was $1 \mathrm{kHz}$ and 1024 samples were taken in each measuring point. Traversing step of the velocity probe was $2 \mathrm{~mm}$ and shifting of the probe was provided by the automatic ISEL 3D traverse system (ISEL, Eiterfeld, Germany).

The turbulence intensity was calculated as follows:

$$
T u=\frac{u_{r m s}^{\prime}}{\bar{U}} \cdot 100(\%)
$$

where $u_{r m s}^{\prime}$ was determined as the root mean square value from the turbulent velocity samples $u$ ' recorded in one measuring point.

The uncertainty of the velocity measurement was calculated according to [3]. The uncertainty for the average velocity of $10 \mathrm{~m} / \mathrm{s}$ was $3.1 \%$. The major source of uncertainty is the calibration process, other sources, such as linearization, A/D conversion, probe positioning, temperature variation of a wire and of ambient air, variation of ambient pressure and humidity have lower effect, but were included in the calculation of the velocity uncertainty.

The uncertainty of the flow rate measurement was calculated according to EN ISO 5167-2. The flow rate coefficient, piping dimensions, expansion coefficient, orifice dimension, pressure difference measurement, and density measurement were taken into account. The type B uncertainty of flow rate measurement reached $3.4 \%$. Repeatability of the flow rate setting, which depends mainly on the ventilator power fluctuations, was experimentally verified. Ventilator power is set by the voltage on the electric power supply. Setting of the flowrate was repeated 15 times and recorded. The standard deviation calculated from this data set, which corresponds to type A uncertainty, reached $0.230 \mathrm{~m}^{3} / \mathrm{h}$. The combined uncertainty of the flow rate was then $2.761 \mathrm{~m}^{3} / \mathrm{h}$. 


\section{Results and discussion}

The numerical simulation provides detailed spaceresolved information about the flow in the channel, while the CTA is only capable of a pointwise measurement. Therefore the axial velocity is displayed in the whole measuring cross-section (Figure 4) based on the simulation data, but only three horizontal and five vertical measuring lines can serve for the direct comparison of CFD and experiments (Figures 5 to 8).

Figure 4 shows the axial velocity component in the measured cross-section with lines on which the experimental measurement was carried out. It is obvious that there are no disturbances in the flow and almost ideally developed flow appears there.

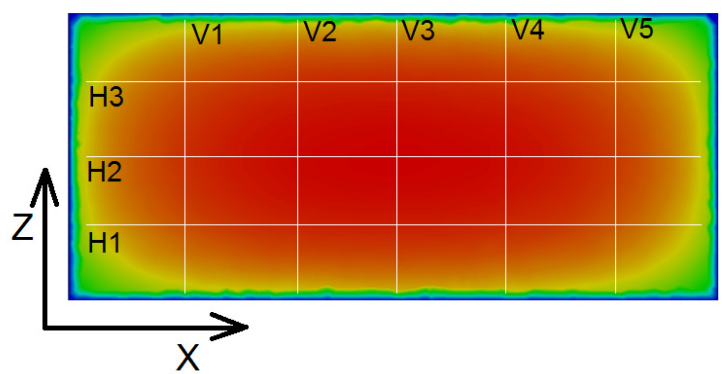

Figure 4. The mean axial velocity component calculated by CFD in the measured cross-section. The white lines correspond with the measuring lines and the labels correspond with labelling in Figures 5 to 8.

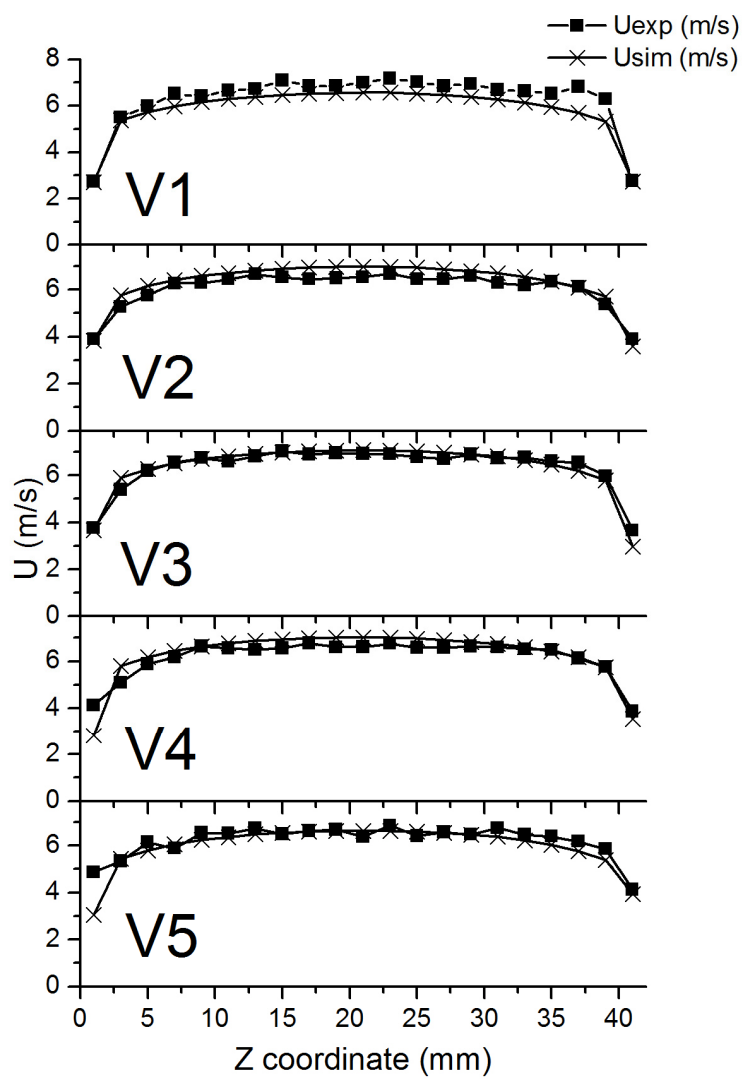

Figure 5. Axial velocity profiles in the vertical measuring lines. The top graph represents the left measuring line in the streamwise view on the measured cross-section.
Comparison of the velocity on the middle vertical measuring line V3 (Figure 5) shows very good agreement of CFD and experiments. Some slight differences can be seen in the V4 and V5 lines close to the probe insertion hole (position $Z=0 \mathrm{~mm}$ ). These differences could arise from the vicinity of the hole, which can cause flow disturbances. These disturbances were not present in the CFD, as no holes were modelled.

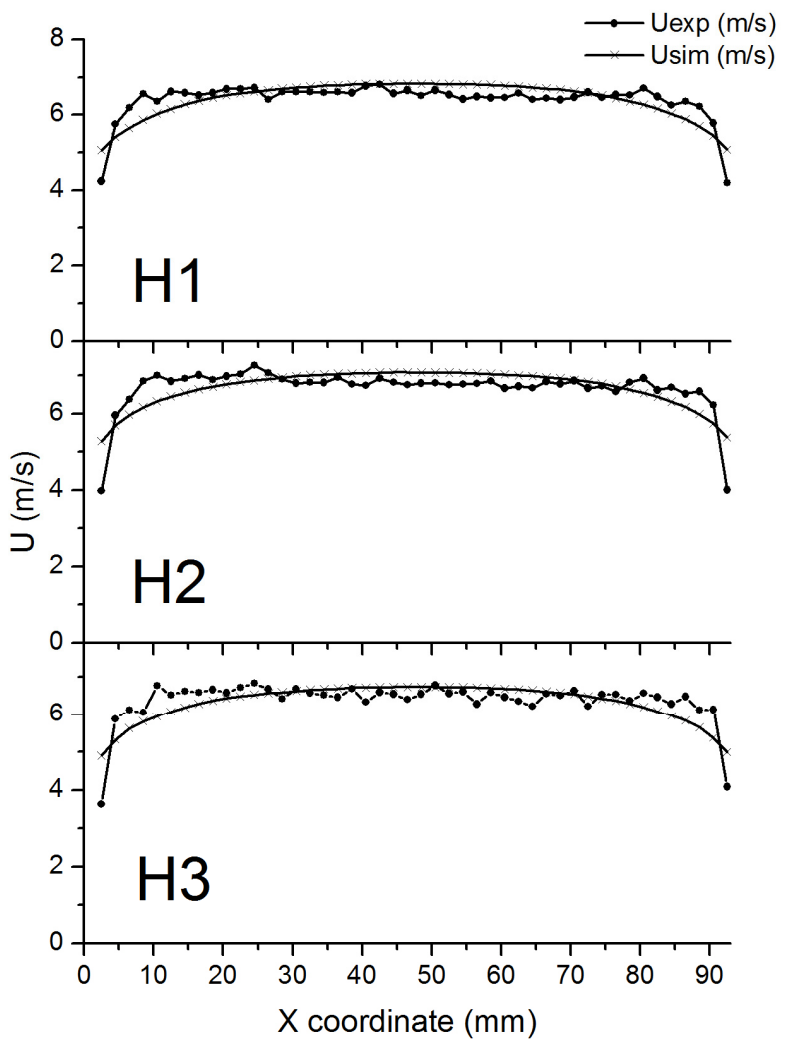

Figure 6. Axial velocity profiles in the horizontal measuring lines. The top graph represents the lowest measuring line.

It should be noted, that the bent probe was used and therefore the heated wire was approximately $5 \mathrm{~mm}$ upstream of the insertion holes plane and therefore only limited effect of the holes was expected. Only minor deviations can be observed in measuring lines V1 and V2.

Horizontal scans (Figure 6) differ significantly. Experimentally acquired profiles appear more turbulent than the numerically simulated. As the flowrate is identical in both the cases, the logical explanation is, that the numerical simulation does not capture the realistic development of the turbulence.

This assumption is partially supported by the comparison of the turbulence intensity presented in Figures 7 and 8. However, as was already mentioned, underestimation of the turbulence intensity in CFD was expected due to the invalidity of the isotropic turbulence assumption of the turbulence model and the geometry simplifications. Apart from that, the turbulence intensity profiles differ in the vertical lines in the vicinity of the insertion holes, which supports the supposition, that the holes could have influenced the measurement close to $\mathrm{Z}=0 \mathrm{~mm}$ coordinates. The cause of asymmetry of the 
turbulence intensity profiles $\mathrm{H} 1$ and $\mathrm{H} 3$ is not known. The additional measurement will be performed to verify that the first measurement was correct. However, the result of the repeated measurement is not available at the time of finalizing this contribution.

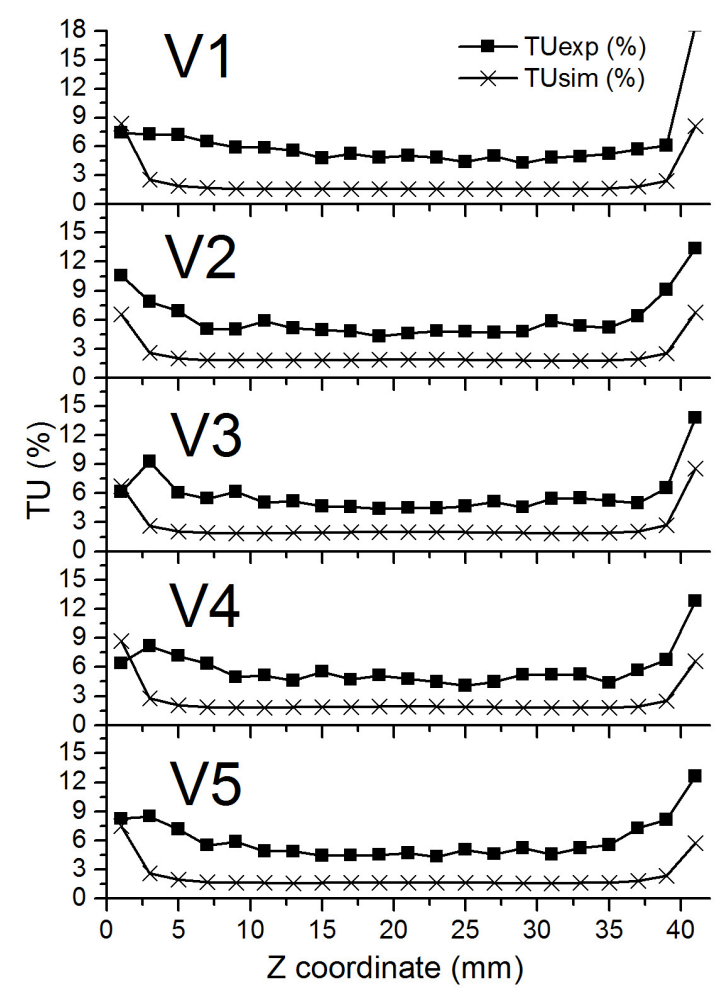

Figure 7. Turbulence intensity in the vertical measuring lines. The top graph represents the left measuring line in the streamwise view on the measured cross-section.

\section{Conclusions}

The comparison of experimentally and numerically acquired axial velocity and turbulence intensity at the boundary of a main section of our measuring rig showed, that despite the simple geometry and general agreement of the average cross-sectional velocity, certain difference was found in the shape of the velocity profile. The difference can be attributed to inaccurately simulated turbulence. Two reasons can be found, that may have influenced that. The first is the isotropic turbulence assumption of the k- $\omega$ SST model. However, more probable explanation is the second one, i.e. that the lower turbulence intensity in the simulation was caused by the absence of geometrical details, such as channel pieces connections, wall roughness and imperfections of the wall surface. These disturbances are transferred in the real flow and affect the development of the turbulence.

Our study indicates that researchers should resist the commonly encountered inclination to restrict the experimental measurement of boundary conditions and the verification of CFD results. It is important to combine both the approaches to achieve valuable results.

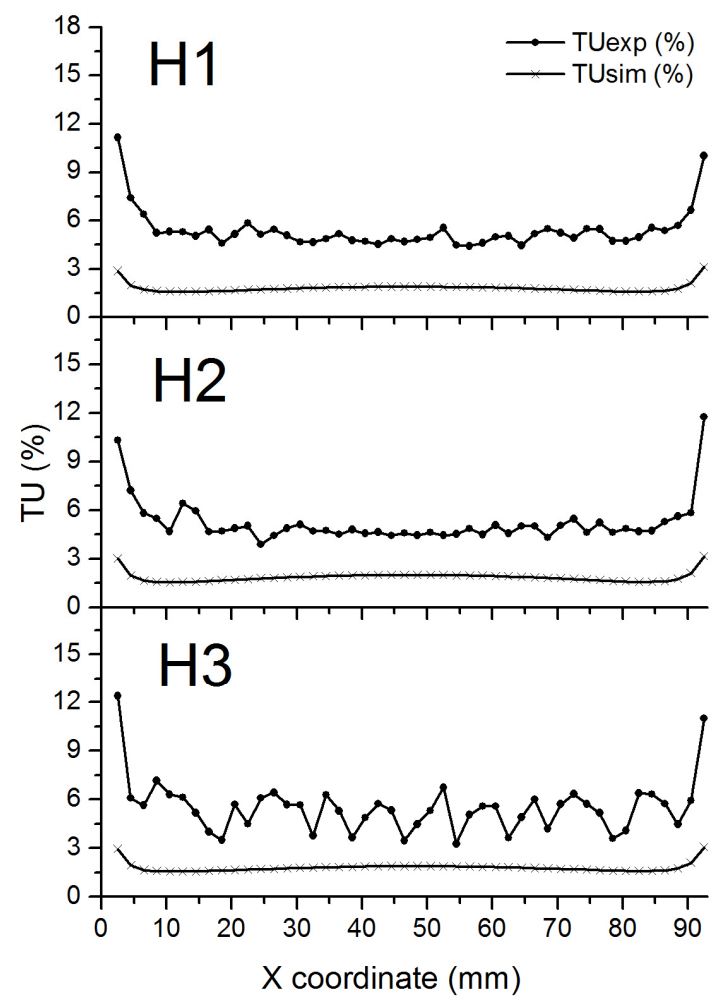

Figure 8. Turbulence intensity in the horizontal measuring lines. The top graph represents the lowest measuring line.

\section{Acknowledgement}

The presented research was supported by the project LO1202 NETME CENTRE PLUS with the financial support from the Ministry of Education, Youth and Sports of the Czech Republic under the "National Sustainability Programme I" and from the project reg. number FSI-S-14-2355 of the Brno University of Technology.

\section{References}

1 L. Šeda, Master Diploma Thesis, Brno University of Technology, 2015.

2 F. R. Menter, AIAA journal 32 (8), 1598 (1994).

3 F. Jorgensen, How to measure turbulence with hotwire anemometers - a practical guide. (Dantec Dynamics, Skovlunde, Denmark, 2002). 